\title{
Study protocol: randomized controlled trial of opioid-free vs. traditional perioperative analgesia in elective orthopedic surgery
}

\author{
Elaine Z. Shing ${ }^{1^{*}}$ (D), Daniel Leas ${ }^{2}$, Caleb Michalek ${ }^{3}$, Meghan K. Wally ${ }^{1}$ and Nady Hamid ${ }^{4}$
}

\begin{abstract}
Background: The medical community is beginning to recognize the contribution of prescription opioids in the growing national opioid crisis. Many studies have compared the safety and efficacy of alternative analgesics to opioids, but none utilizing a completely opioid-free perioperative protocol in orthopedics.

Methods: We developed and tested an opioid-free perioperative analgesic pathway (from preoperative to postoperative period) among patients undergoing common elective orthopedic procedures. Patients will be randomized to receive either traditional opioid-including or completely opioid-free perioperative medications. This study is being conducted across multiple orthopedic subspecialties in patients undergoing the following common elective orthopedic procedures: single-level or two-level ACDF/ACDA, 1st CMC arthroplasty, Hallux Valgus/Rigidus corrections, diagnostic knee arthroscopies, total hip arthroplasty (THA), and total shoulder arthroplasty/reverse total shoulder arthroplasty (TSA/RTSA). The primary outcome measure is pain score at $24 \mathrm{~h}$ postoperatively. Secondary outcome measures include pain scores at additional time points, medication side effects, and several patientreported variables such as patient satisfaction, quality of life, and functional status.
\end{abstract}

Discussion: We describe the methods for a feasibility randomized controlled trial comparing opioid-free perioperative analgesics to traditional opioid-including protocols. We present this study so that it may be replicated and incorporated into future studies at other institutions, as well as disseminated to additional orthopedic and/or non-orthopedic surgical procedures. The ultimate goal of presenting this protocol is to aid recent efforts in reducing the impact of prescription opioids on the national opioid crisis.

Trial registration: The protocol was approved by the local institutional review board and registered with clinicaltrials.gov (Identifier: NCT04176783) on November 25, 2019, retrospectively registered

Keywords: Pain management, Opioid crisis, Elective surgery

\footnotetext{
*Correspondence: elaine.shing@atriumhealth.org

${ }^{1}$ Carolinas Medical Center, Atrium Health Musculoskeletal Institute, P.O. Box 32861, Charlotte, NC 28232, USA

Full list of author information is available at the end of the article
}

(c) The Author(s). 2021 Open Access This article is licensed under a Creative Commons Attribution 4.0 International License, which permits use, sharing, adaptation, distribution and reproduction in any medium or format, as long as you give appropriate credit to the original author(s) and the source, provide a link to the Creative Commons licence, and indicate if changes were made. The images or other third party material in this article are included in the article's Creative Commons licence, unless indicated otherwise in a credit line to the material. If material is not included in the article's Creative Commons licence and your intended use is not permitted by statutory regulation or exceeds the permitted use, you will need to obtain permission directly from the copyright holder. To view a copy of this licence, visit http://creativecommons.org/licenses/by/4.0/ The Creative Commons Public Domain Dedication waiver (http://creativecommons.org/publicdomain/zero/1.0/) applies to the data made available in this article, unless otherwise stated in a credit line to the data. 


\section{Background and rationale}

Opioids have long been used in various forms for pain control in the medical field. While there is demonstrated analgesic effect of these compounds [1], they are also associated with a number of side effects, including constipation, nausea/vomiting, hyperalgesia [2, 3], delirium [4], opioid dependence/withdrawal, and even respiratory depression/death [5]. Their use for acute pain management has undergone a logarithmic increase in the past twenty years, which has also brought a concomitant rise in opioid-induced side effects. Patient expectations of opioid medications has driven a rapid rise in outpatient opioid prescriptions for both short and long-acting opioids [6], which have additionally shown substantial addiction potential. It has been found that two-thirds of patients taking opioids 3 months after elective surgery are still on opioids at an average of 4.8 years later [6].

The existing literature on opioid prescribing for musculoskeletal conditions has consistently demonstrated frequent over-prescribing, leading to unused pills available for nonmedical use or diversion [7-12]. These prescriptions have become a source of significant mortality in the United States, with nearly 48,000 opioid-related overdose deaths in 2018 alone (illicit and prescription combined) [13]. However, the rate of prescription-opioid deaths encouragingly dropped for the first time in nearly 10 years, decreasing from 17,000 to 15,000 deaths between 2017 and 2018 [13].

This decrease in prescription-opioid death rates can largely be attributed to widespread efforts to reduce provider reliance on opioids. The scientific community now has an improved understanding of the risks and benefits of various forms of pain medication. Efforts have been made to identify synergistic compounds to use for acute pain management in the perioperative time period, all of which report some degree of opioid-sparing effects. These studies have focused on the safety and efficacy of gabapentinoids [14-16], local administration of sodiumchannel blockers such as lidocaine and bupivacaine [17-19], opioid-free anesthesia [20-22], cryotherapy [23, 24], dexamethasone $[25,26]$, and non-steroidal anti-inflammatories (NSAIDs) and acetaminophen [27-33].

The most robust adjuvant effects have been reported with NSAIDs and acetaminophen. Some studies indicate that there may be a synergistic effect between these two to reduce postoperative opioid intake [34]: oxycodone $15 \mathrm{mg}$ and ibuprofen $600 \mathrm{mg}$ have comparable treatment efficacy as measured by number needed to treat (NNT) ( 2.3 vs. 2.4 , respectively). Ibuprofen $800 \mathrm{mg}$ has the highest treatment efficacy (NNT 1.6) of many reported analgesic combinations [35].

At this time, no study has examined the possibility of utilizing a multi-modal perioperative analgesic pathway that does not include some form of opioid medication in orthopedic patients. We report an ongoing feasibility randomized controlled trial (RCT) designed with the objective to investigate the safety and efficacy of opioidfree pain management in elective orthopedic procedures and determine if opioid-based analgesia is needed in orthopedic surgery. This is, to our knowledge, the first investigation of its kind. Patients in the opioid-free analgesia arm did not receive any opioid medications in the pre-, intra-, and postoperative phases through the time they followed up in clinic (including the general and local anesthesia protocols). This article describes our opioid-free clinical pathway in detail with review of the existing literature to justify each component. The ultimate goal is that this protocol may be replicated for future studies and potentially incorporated into a perioperative clinical pathway. Should this study demonstrate equivalent efficacy of opioid-free and opioid-based analgesic protocols, then its findings may also help reduce the amount of prescribed opioid medications for patients undergoing elective orthopedic surgery.

\section{Methods: trial design, patient selection, and intervention \\ Overview}

This parallel group RCT was performed at a single institution, which is a large private orthopedic practice with an affiliated research institute, across 6 subspecialties (Shoulder \& Elbow, Hip \& Knee, Hand, Sports, Spine, Foot \& Ankle). Specifically, surgeons from this one practice enrolled their patients; patients had surgery across six facilities that were either hospital-affiliated or private ambulatory surgery centers (Atrium Health Mercy, Charlotte Orthopedic Hospital, Carolinas Medical Center One-Day Surgery, Charlotte Surgery Center, Mallard Creek Surgery Center, and Matthews Surgery Center). The study also included an observational arm for those patients who did not wish to be randomized. The protocol was approved by the local institutional review board and registered with clinicaltrials.gov (Identifier: NCT04176783; Protocol version 7; October 11, 2019), retrospectively registered (URL: https://clinicaltrials.gov/ct2/show/NCT04176 783?term=NCT04176783\&draw=2\&rank=1). Figure 1 provides a summary of the clinical pathways.

A draft medication protocol was developed for each subspecialty, covering preoperative, intraoperative, postanesthesia care unit (PACU), postoperative, and discharge settings based on the literature. A steering committee consisting of physicians, anesthesiologists, nurses, and the research team was created to develop these protocols. Specifically, one orthopaedic surgeon was included from each of the following subspecialties: Shoulder \& Elbow, Spine, Foot \& Ankle, Hand, Sports, and Hip \& Knee. One orthopaedic surgery resident was included. Finally, four anesthesiologists to represent both hospital systems were 


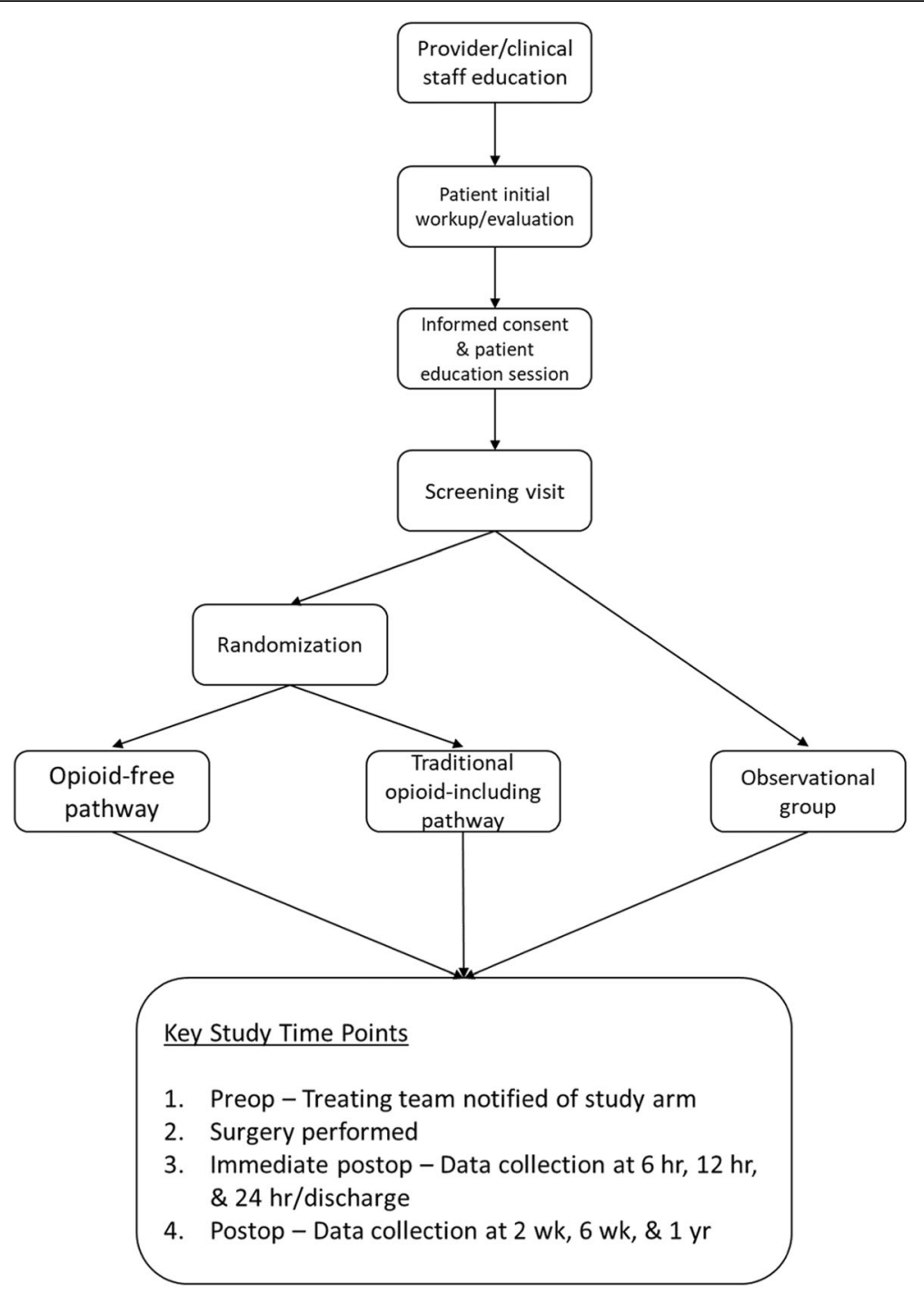

Fig. 1 Flow diagram illustrating study protocol

on the steering committee. These steering committee members are experts in their respective fields and utilized existing literature (summarized in the background section) to inform the inclusion of various non-opioid analgesics in the protocols. Pilot testing was performed on a group of shoulder arthroplasty patients (data published previously in a case series [36]). The final protocols are presented in Table 2. While the choice of each medication in these protocols was informed by the literature [14-35, 37] and expert consensus of the steering committee, the proposed study will assess the efficacy of these protocols for pain control in a larger group of elective orthopedic surgery patients.

Patients were randomized to one of two arms. The treatment group included treatment without the use of opioids. The control group included a traditional opioid- based pain protocol, agreed upon by the treating surgeons. The observational group consisted of patients who were eligible for the study but were not willing to be randomized to a postoperative pain management pathway. They were treated based on patient/surgeon preference and included in an associated cohort study.

The study population consisted of patients aged $\geq 18$ undergoing the following routine elective orthopedic surgical procedures: single-level or two-level Anterior cervical discectomy and fusion/Anterior cervical disc arthroplasty (ACDF/ACDA), 1st carpometacarpal (CMC) arthroplasty, Hallux Valgus/Rigidus corrections, diagnostic knee arthroscopies, total hip arthroplasty (THA), and total shoulder arthroplasty/reverse total shoulder arthroplasty (TSA/RTSA). Eligible patients who provided informed consent were randomized to one of the two study arms. 
Outcomes were assessed at multiple time points $-6,12$, and $24 \mathrm{~h}$ postoperatively, then 2 weeks, 6 weeks, and 1 year postoperatively.

\section{Care team education}

A key element of the clinical protocol was providing physicians and clinical/research staff with education on opioid medications and their role in perioperative painrelief. Studies have identified knowledge gaps in providers managing chronic [38] and acute postoperative pain [39]. However, provider-directed education on opioid-related issues can be successful in decreasing postoperative opioid use [40-42]. As such, institutions are beginning to recommend interventions for providers regarding these subjects [22]. Our study employed multiple in-service sessions for investigators, research staff, and nurses/hospital support staff to educate and prepare them for the opioid-free clinical pathway. These sessions outlined the purpose of the study, highlighted the dangers and pitfalls of opioid use, and provided evidence for pharmacologic alternatives. We recognized that this pathway represented a significant change to the typical work-flow for many care team members including anesthesiologists, nurses, and surgeons. We anticipated skepticism among some health care workers and found that pre-study meetings and in-service training sessions helped to offer clarity about the specifics of the clinical pathway and instilled confidence that patients' comfort levels would remain a high priority.
Patient selection, recruitment, and randomization Eligibility and recruitment

Eligibility was determined based on the inclusion and exclusion criteria listed in Table 1. Eligible participants were identified after initial evaluation by a staff physician, determined to require one of the qualifying procedures, and approached for informed consent by the physician and research staff. At this time, patients watched an introductory video explaining the purpose of the clinical trial. The physician also led an in-depth preoperative discussion explaining each approach to analgesia (opioid vs. opioidfree) and postoperative expectations. The inclusion of this process was driven by recent data showing decreased use of postoperative opioid medications after patients received preoperative opioid education [43-46]. If patients declined to participate in the randomized trial, they were presented with the option of enrolling in the observational group where they would be able to select either the control opioid-containing arm or the opioid-free arm. There were no restrictions on patient care due to enrollment in the study.

\section{Screening visit}

Participants underwent an initial screening visit that included a series of questionnaires (dependent upon the type of surgery being performed) and physical exam. The questionnaires, described in the outcomes section below, asked about level of pain, function, and satisfaction with pain control. The exam included an assessment of

Table 1 Eligibility Criteria

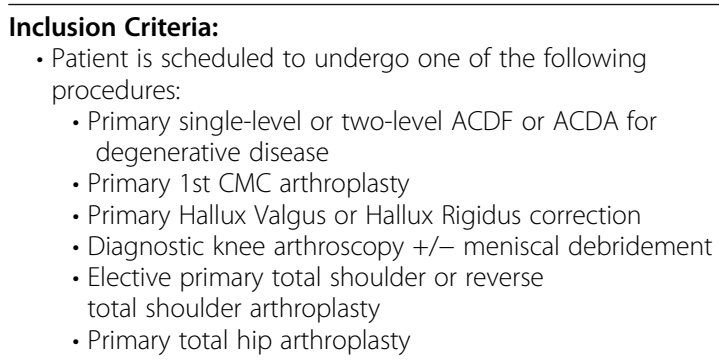

Exclusion Criteria:

- Revision surgery for one of the study-specific procedures

- Chronic opioid therapy - per investigator discretion

- Significant liver disease - (NOTE: Patients with a history of liver disease had a hepatic panel drawn that was reviewed by the study investigator to assess if the values were within acceptable limits for inclusion in the study)

- Fracture or soft tissue injury

- Sickle cell disease

- Workers compensation

- Alcohol dependence

- Contra-indication to regional anesthesia

- History of gastrointestinal (Gl) bleeding or peptic ulcer

- History of bleeding problems

- Patients taking anticoagulants, not including aspirin (only applied to Randomized portion of study. These patients could still participate in Observational Control Group)

- Renal insufficiency - Creatinine clearance less than $30 \mathrm{~mL} /$ min (only applied to patients having surgery requiring NSAID treatment)

- Hammertoe in isolation (Hallux Valgus/Rigidus exclusion only)

- Concomitant meniscal repair or microfracture (Knee Arthroscopy exclusion only)

- Ineligible for spinal anesthesia (THA exclusion only)

- Previous ipsilateral hip surgery, not including hip scope (THA exclusion only)

- Allergy to non-steroidal anti-inflammatory medications (NSAIDs) 
range of motion, when applicable. All patients were asked to complete a Resilience Questionnaire (Resilience Scale 5 , [47]) at this visit.

All patients underwent a blood draw $(5 \mathrm{ml})$ to confirm eligibility based on creatinine clearance $(\mathrm{CrCl})$, which was calculated with the Cockcroft-Gault Formula. Patients with a $\mathrm{CrCl}<30 \mathrm{~mL} / \mathrm{min}$ were excluded from the study. Patients with $\mathrm{CrCl}$ between 30 and $60 \mathrm{~mL} /$ min received a half-dose of NSAIDs if enrolled in the intervention arm of the study. Patients with reported liver disease received a second blood draw to evaluate hepatic liver enzymes and ensure safe dosing of acetaminophen.

\section{Randomization}

Patients were randomized in a 1:1 fashion to one of the treatment groups - Control (with opioids) or Intervention (opioid-free). Randomization occurred within 1 week prior to surgery and was performed with a random number generator in REDCap by research staff [48]. This random number generator was built into the REDCap system such that the study coordinators and investigators were unaware of what the randomization schedule would be. The allocation was then communicated to the surgical team via email. A randomization form was placed on the patient chart prior to surgery. Patients were not blinded to the treatment arm.

Once randomized to an arm, the treating team of surgeon, anesthesiologist, and clinical staff were notified of the result. There was no blinding involved in this study. Patients were identified as being a part of the study, and the corresponding treatment arm was listed in their hospital chart. Reminders of each patient's treatment designation were provided to the anesthesiology team in the preoperative holding area on the day of surgery.

\section{Sample size calculation}

Estimated sample size was calculated using data from a previous study comparing the pain numeric rating scale (NRS) at $24 \mathrm{~h}$ between patients receiving opioid medications or not [36]. This was a noninferiority design with a 2-point margin. The mean pain score for the traditional group was 3.2 and the mean pain score for the nonopioid group was 2.5 with a pooled standard deviation of 2.5. An alpha level of 0.5 and a minimum power of $80 \%$ yielded 50 patients per group per procedure. Therefore, the target sample size was 300 completed patients at 24 $\mathrm{h}$ in the traditional group and 300 completed patients at $24 \mathrm{~h}$ in the opioid-free group.

\section{Intervention}

Patients in the opioid-free treatment arm did not receive any opioid medications from the preoperative period to the postoperative period. The control arm included traditional opioid-based analgesia. Medications in the treatment arm were selected based on findings from current literature on opioid-alternatives. Medications in the control arm were included as usual perioperative analgesic options. Some variations to the treatment pathways existed by procedure type. Tables 2 and 3 illustrate the full protocol of analgesic administration for each arm. As described above, the steering committee developed the protocols based on peer-reviewed literature and expert consensus among the committee.

We employed several strategies to improve adherence to study protocols. First, we took measures to ensure that medical staff were clearly aware of which study arm each patient was enrolled in. The treating surgeon and key staff contacts at the surgery location's preoperative, anesthesia, post anesthesia care, and postoperative units received an email notification with the patient's assigned study arm at the time of randomization (1 week prior to surgery) and 2 days prior to the surgery. This email also contained a reminder of the medication protocol specific to that case. A notification sheet and copy of the medication protocol was placed on the front of the patients' hospital charts along with an opioid-free wristband if the patient was randomized into the opioid-free arm of the study. Secondly, all patients received a diary after their surgery to record medication usage in the first 2 weeks of the postoperative period. This diary helped monitor protocol adherence, at least in self-reported form.

Importantly, patients and care team members were also free to communicate with the treating physician any levels of discomfort found to be unsatisfactory to the patient. If a patient had exhausted all options in the opioid-free pathway, they were given the option to receive opioids. Upon discharge, patients were given instructions to contact their physician for unacceptable levels of pain to receive an outpatient prescription of an opioid. However, no "rescue" or "just in case" prescriptions were given to patients upon discharge to avoid prescription diversion and unnecessary home stores of opioids. Additionally, if a patient deviated from the medication protocol assigned to them, they were not considered study failures. Instead, we continued to follow the patient through study completion and continued to track patient-reported outcomes and total opioid consumption.

\section{Methods: data collection and outcome measures Baseline assessments and frequency of follow-up assessments}

A full list of assessments obtained at each time point is summarized in Table 4.

We obtained baseline assessments during each patient's screening visit. These measures included, but were not limited to, demographics, comorbidities, subjective pain ratings, constipation ratings, patient reported 


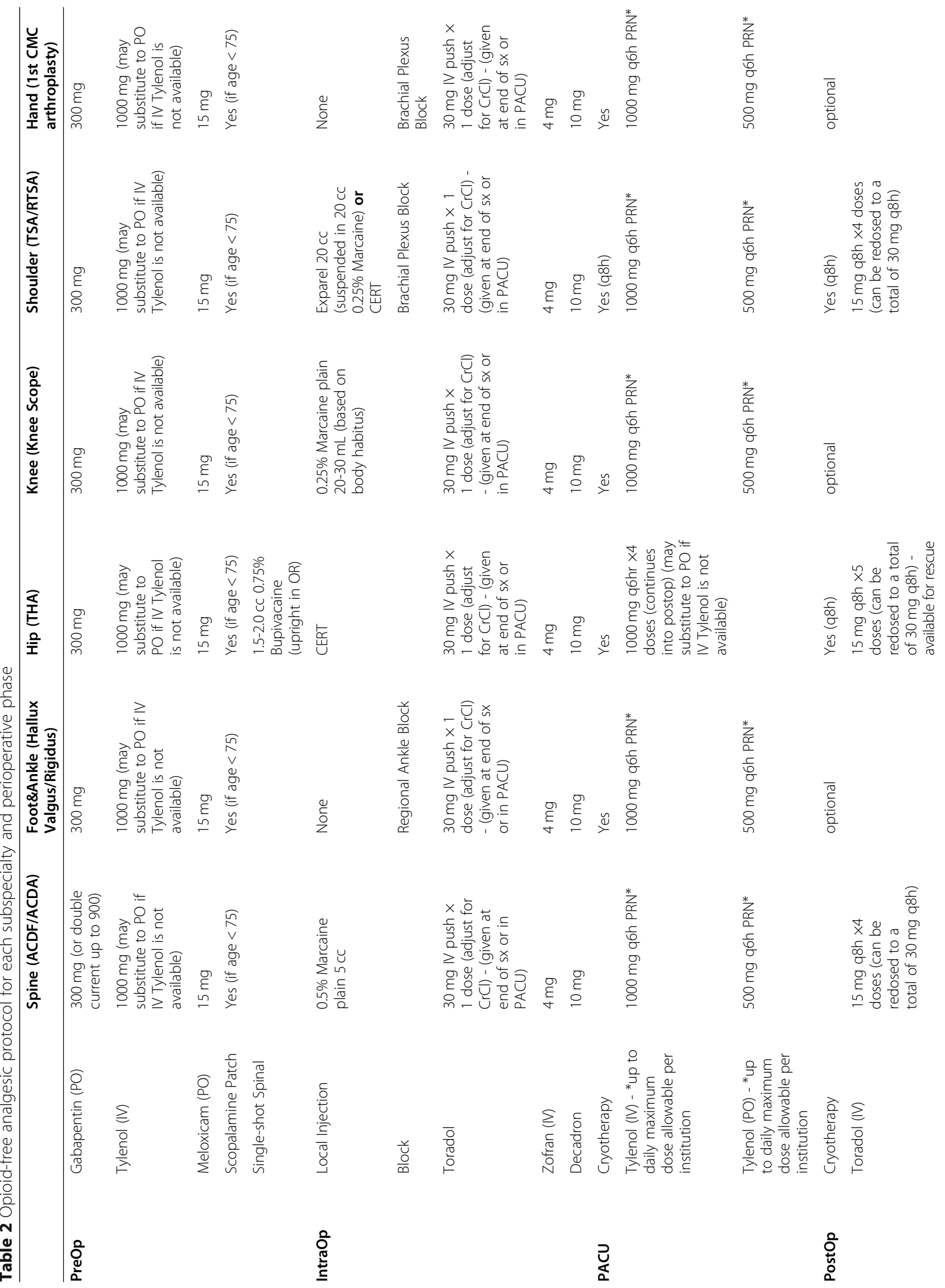




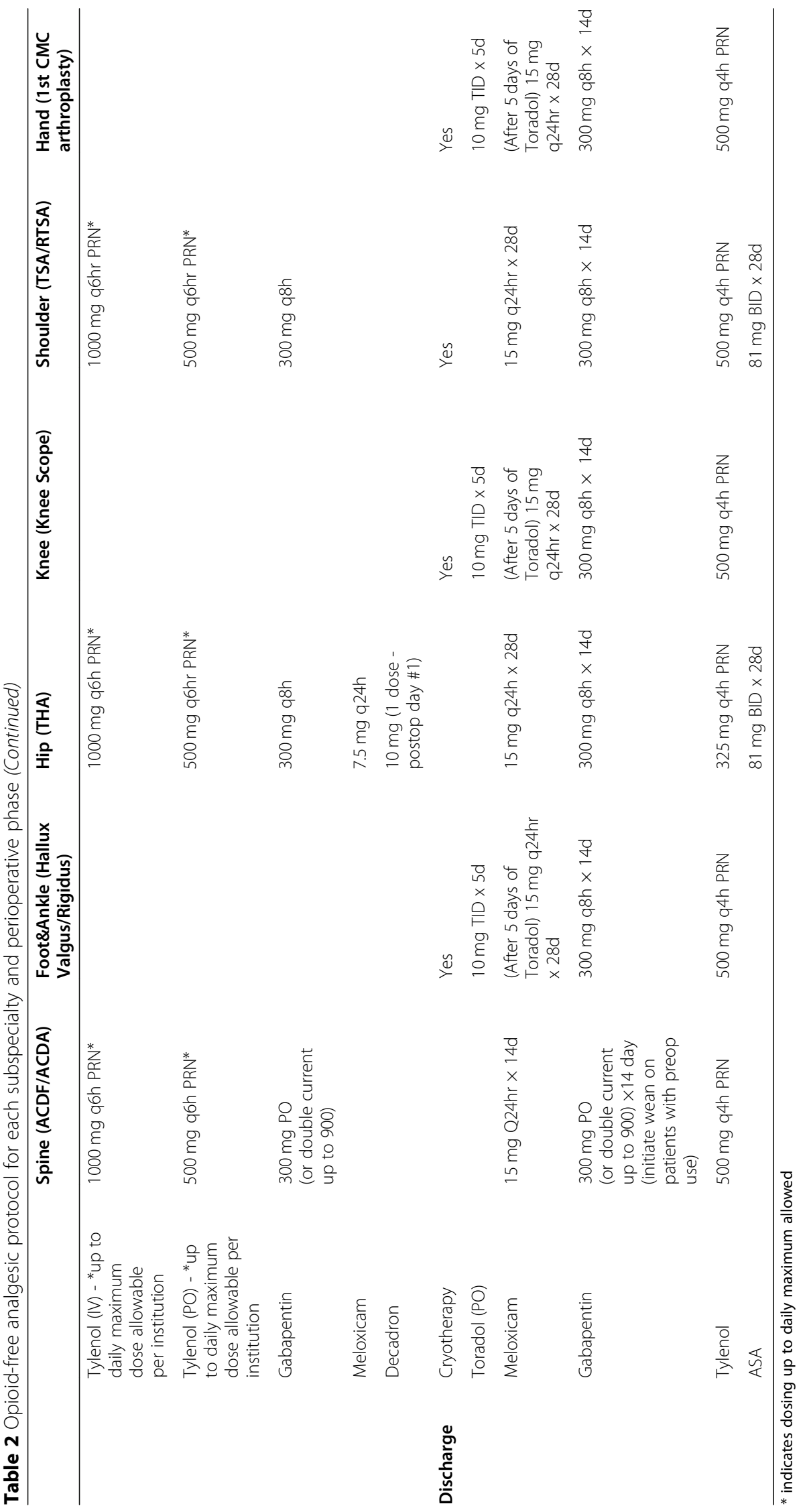




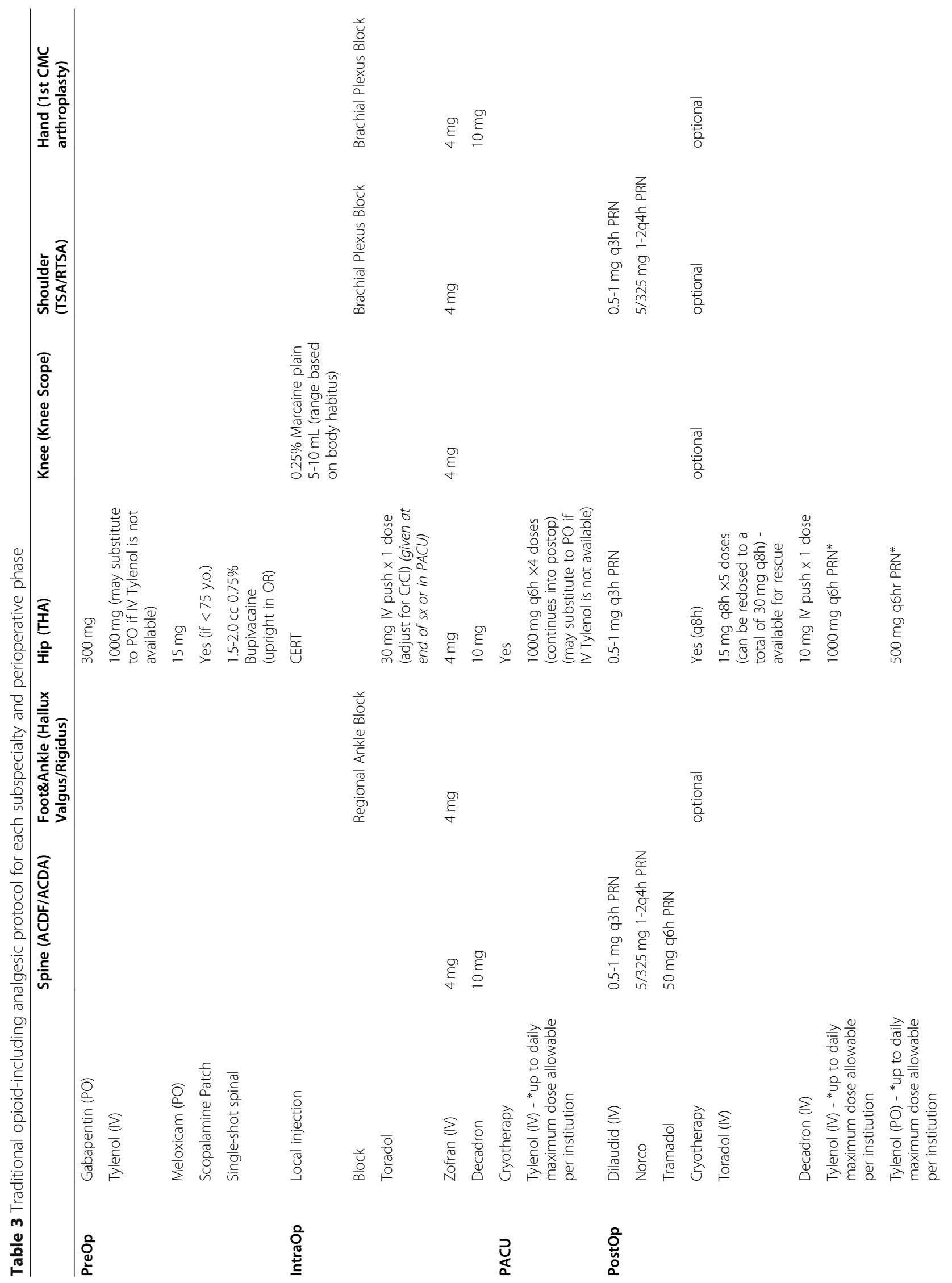




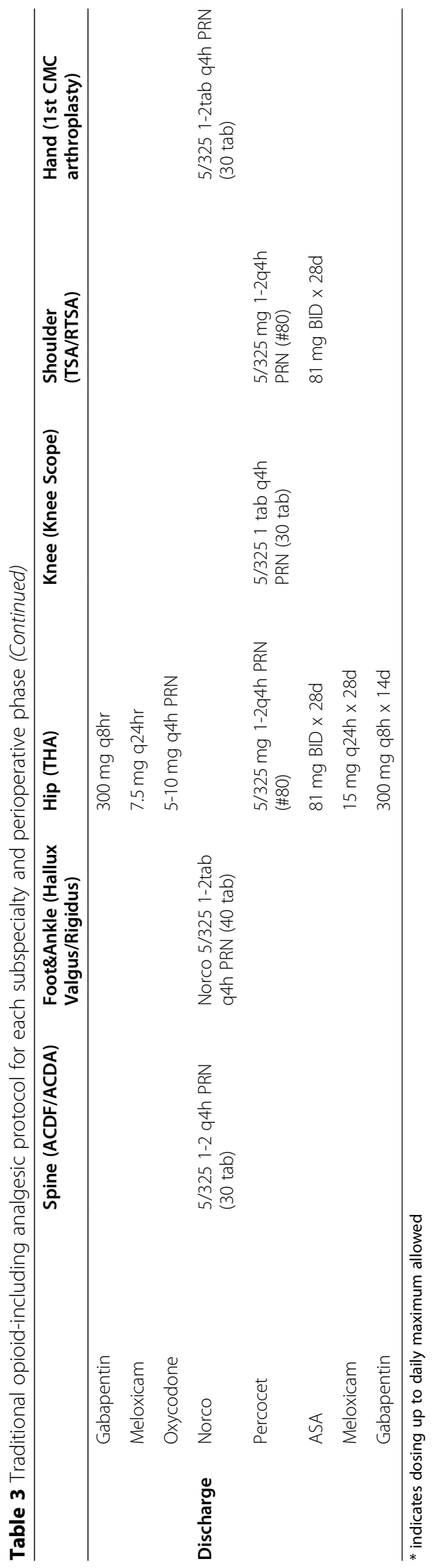


Table 4 Full list of assessments obtained at each time point

\begin{tabular}{|c|c|c|c|c|c|c|c|c|}
\hline Procedure & Preop/Screen & Op & $6 \mathrm{~h}$ & $12 \mathrm{~h}$ & $24 \mathrm{~h}$ & 2 week & 6 week & 1 year \\
\hline ICF & $x$ & & & & & & & \\
\hline Demographic & $x$ & & & & & & & \\
\hline Comorbidities & $x$ & & & & & & & \\
\hline $\begin{array}{l}\text { Resilience Questionnaire } \\
\text { (RS-5) }\end{array}$ & $x$ & & & & & & & \\
\hline Central Sensitization Inventory & $x$ & & & & & & & \\
\hline Creatinine Clearance & $x$ & & & & & & & \\
\hline Randomization & $\begin{array}{l}\mathrm{X} \\
\text { (within one week prior to surgery) }\end{array}$ & & & & & & & \\
\hline Complications (nausea, constipation, falls) & & & $x^{a}$ & $x^{a}$ & $x^{a}$ & $x$ & $x$ & $x$ \\
\hline Delirium Score $(C A M 25)^{a}$ & & & $x^{a}$ & $x^{a}$ & $x^{a}$ & & & \\
\hline Patient Comfort Level (NRS) ${ }^{a}$ & & & $x^{a}$ & $x^{a}$ & $x^{a}$ & & & \\
\hline $\begin{array}{l}\text { ConMeds (specifically anti-emetic/nausea } \\
\text { and pain medication) }\end{array}$ & $x$ & $x$ & $x^{a}$ & $x^{a}$ & $x^{a}$ & $x$ & $x$ & $x$ \\
\hline Surgical \& Hospital-Stay Information & & $x$ & & & & & & \\
\hline Pain Score (NRS), current & $x$ & & $x$ & $x$ & $x$ & $x$ & $x$ & $x$ \\
\hline Pain Score (NRS), average & $x$ & & & & & $x$ & $x$ & $x$ \\
\hline Constipation Questionnaire (PAC-SYM) & $x$ & & & & & $x$ & $x$ & $x$ \\
\hline $\begin{array}{l}\text { Patient Reported Outcomes Questionnaires } \\
\text { (specific to applicable surgery) }\end{array}$ & $x$ & & & & & $x$ & $x$ & $x$ \\
\hline Patient Pain (NRS) Diary \pm & & & & & & $x$ & & \\
\hline Patient Comfort (NRS) Diary \pm & & & & & & $x$ & & \\
\hline Patient Pain Medication Diary \pm & & & & & & $x$ & & \\
\hline $\begin{array}{l}\text { Patient Collar Compliance Diary } \pm \\
\text { (ACDF/ACDA patients only) }\end{array}$ & & & & & & $x$ & & \\
\hline
\end{tabular}

${ }^{a}$ Only applies to inpatient procedures

\pm The diaries will be completed daily from day of discharge until the 2 week visit. They will be collected at the 2 week visit

outcomes specific to the planned procedure, and resilience ratings.

Initial follow-up assessments were obtained at 6, 12, and $24 \mathrm{~h}$ after surgery. Patients were asked to return for postoperative follow-up visits at 2 weeks, 6 weeks, and 1 year after surgery. These visits were scheduled to be at routine visits that were already a part of our investigators' standard-of-care treatment time tables. Patients that were unable to attend any in-person visit were asked to completed all study patient-reported outcomes via phone call or REDCap electronic survey for that visit to facilitate retention and optimal follow-up. Pain level, medication usage, constipation level, complications, and patient reported outcomes were gathered during these visits.

Each patient received a diary upon hospital discharge to record their pain levels, overall comfort/satisfaction levels, and medication usage. Patients who underwent ACDF/ ACDA surgery were also asked to record their compliance with soft collar equipment. Information from the diaries was collected at the 2-week postoperative visit.

\section{Primary outcome measures}

The primary outcome measure was pain at 24-h postoperatively. If the patient was in-hospital, the pain rating was obtained by the nurse. If the patient had been discharged, they were called by research staff to obtain a pain rating. This outcome was measured on a 0-10 NRS, a reliable and valid measure of present-moment subjective pain [49].

\section{Secondary outcome measures}

Secondary outcomes of interest included pain ratings (NRS) at the additional time points noted previously as well as other clinical and patient-reported variables.

Basic clinical information was collected at time of surgery or hospitalization, including length of hospital stay, intraoperative complications, and length of surgery. Morphine milli-equivalents were recorded in-hospital, and post-operative opioid use was recorded after discharge. Medication side effects were recorded as episodes of delirium, number of falls, and patient reported nausea/constipation. 
Several patient-reported variables were collected throughout the duration of the study and included nausea/constipation, satisfaction with pain control and surgical experience, quality of life, resilience, and functional status specific to the surgery performed. Functional outcomes were assessed with validated, standardized questionnaires (e.g. American Shoulder and Elbow Surgeons Shoulder Score for TSA/RTSA patients [50] and Foot and Ankle Ability Measure for hallux valgus/rigidus patients [51]). We also collected qualitative data from patients in the control group regarding unused opioid pills. They were asked to describe if and how the pills were disposed of or secured away if not disposed.

\section{Covariates and confounders}

Several variables were measured as potential covariates, including pre-operative opioid use, pre-operative pain scores, concomitant procedures, medical comorbidities (based on the Charlson Comorbidity Index), BMI, alcohol or other non-prescribed medication use to help with comfort, tobacco use, and tourniquet time (when applicable). Basic demographic information was also noted.

\section{Methods: data management, analysis, \& additional information}

\section{Data management}

Data were collected by research staff and entered into REDCap (http://project-redcap.org/) [46] on at least a weekly basis. REDCap is a secure web application designed to support data capture for research studies. The program provides audit trails for tracking data manipulation and user activity, as well as automated export procedures for data downloads to common statistical packages (Excel, SPSS, SAS, Stata).

Study information is de-identified by removing PHI and using coded subject identifiers. Study data is kept in locked cabinets/rooms only accessible by research staff. Electronic data is kept in an access-privileged, passwordprotected, encrypted database.

Adverse events were collected as part of the surgical and follow-up source documents. They were collected via patient reporting, questionnaires, and chart review. Serious Adverse Events that were "related," "probably related" or had an "unknown" relatedness to the study procedure were reported via secure email to the data safety monitoring board as they occurred. These occurrences were reviewed every other month or as needed by the data safety monitoring board, which is made up of members of the OrthoCarolina Research Institute (OCRI) Research Advisory Committee. At each meeting, the study was reviewed for adverse events, serious adverse events, and overall feasibility issues. Additionally, the board conducted internal audits for this clinical trial.
Initial auditing occurred after surgery and data collection had been completed for one patient in each study group per subspecialty. After that point, auditing has occurred on an as-needed basis with an internal audit happening at least once per year.

\section{Statistical analysis}

The primary outcome of pain at 24-h postoperatively will be assessed for normality using visualization methods, including QQ plots as well as statistical analysis tests including Shapiro-Wilk. If these data are normally distributed, an independent $\mathrm{t}$-test will be used to compare pain at 24-h postoperatively. If these data are non-normal, a Mann-Whitney $U$ test will be used to compare pain at $24-\mathrm{h}$ postoperatively between treatment groups.

Normality testing as previously described will be evaluated for all continuous secondary outcomes including pain at additional postoperative time points, length of stay, morphine milliequivalents, satisfaction, and scores from patient reported outcome measures. The appropriate statistical test, either independent t-tests or MannWhitney $\mathrm{U}$ tests, will be used to compare these outcomes between treatment groups. Categorical outcomes such as intraoperative complications, medication side-effects, and other postoperative complications, will be compared between treatment groups using a Chi-Square of Fishers Exact test.

Statistical associations between covariates and outcomes will be evaluated using the appropriate statistical methodology as previously described. Multivariable linear regression models will be appropriately fitted to continuous data based on the distributions of that data. Multiple logistic regression models will be fitted for all dichotomous outcome variables. Appropriate variable selection methods and model fit statistics will be used to determine the best fitting model to determine the effects of the treatment after adjusting for significant effects of covariates.

Finally, a per protocol analysis is planned. There is no plan to impute missing data and all statistical analyses will be conducted with the available data. We also plan to include sub-group analyses for the individual subspecialties.

Any protocol amendments were first submitted to the Institutional Review Boards. Upon their approval, the new protocols were disseminated to all research staff (including all investigators, research coordinators, etc.) via email with a summary of all changes. If the amendment required additional training for study procedures, training was conducted with the applicable study staff. If the amendment changed anything about what is reported on clinicaltrials.gov, then the study's registration on clinicaltrials.gov was updated accordingly. 
In the event that a subject was harmed as a result of their participation in this study, the clinical team provided or arranged for treatment as necessary. This treatment, as well as other medical expenses, were billed to the subject or the subject's insurance company in the usual manner. Subjects did not waive any legal rights by signing the informed consent form for this study.

\section{Dissemination}

Once the study has entered the data reporting phase, the datasets used and/or analysed during the current study will be available from the corresponding author on reasonable request. The protocol and results will be released onto clinicaltrials.gov as is required upon study completion. Final study results will be published in a peer-reviewed, PubMed-indexed journal to reach healthcare professionals. The authors will also seek to present the study findings at relevant orthopaedic subspecialty society meetings. A website exists on the OrthoCarolina Research Institute website describing this project. Final findings and results will be published on this website for the general public, as well as disseminated through the OCRI social networking channels.

\section{Discussion}

This study is, to our knowledge, the first to evaluate the safety and efficacy of utilizing a completely opioid-free perioperative analgesia protocol. Studies to date have combined opioid and non-opioid medications or pharmacologic alternatives in the same protocol. This study will be the first to try and distinguish the analgesic effects of opioid-including and opioid-free pain regimens. A particularly novel element of this protocol is the elimination of opioids during the surgical procedure. It is unknown what effect opioid administration during anesthesia has on patients with regards to known opioid-related side effects including post-operative delirium, nausea, and constipation. An early case-series study offers encouraging results. Leas et al. (2019) [36] reported that patients undergoing elective shoulder arthroplasty and treated with non-opioid multimodal analgesia experienced overall low levels of pain at $24 \mathrm{~h}$ after surgery (2.5 out of 10), which remained stable at all postoperative time points. Additionally, there were low rates of reported nausea, constipation, and falls.

Another strength of the present study is the randomized study design, which limits error and bias in the results. Conclusions gained from the RCT are also potentially bolstered by the addition of the prospective observational arm. Finally, the results from this study are generalizable, as we enrolled patients from multiple orthopedic subspecialties, allowing us to draw conclusions that may impact orthopedic surgery as a whole.
The major limitation of this study is the inclusion of a select few orthopedic procedures. All surgeries were relatively straightforward (i.e. no revision surgeries) and commonly performed procedures. Patients who are optimal candidates for these elective surgeries may be healthier, less likely to experience post-operative complications, and/or more likely to be opioid-naïve than other orthopedic populations (e.g. trauma or oncology patients). Complicated procedures may also inherently be experienced as more painful. The orthopedic community will need to establish the efficacy of opioid-free multimodal analgesic protocols in a broader range of patients and procedures, including complex operations and patients with significant medical comorbidities.

We recognize that there are some substantial barriers to establishing completely opioid-free analgesic pathways in practice. When pain was introduced as the fifth vital sign in the 1990s, a cascade of events commenced that resulted in unintended consequences of overzealous treatment of pain. Clinicians started relying on opioid medication to eliminate patients' pain, and public misconceptions about the efficacy of opioids for treating pain grew [52, 53]. Partially, the underlying problem was a mounting lack of understanding about the risks and benefits of opioid medications. Our study attempts to address these roadblocks by providing both patients and clinicians with educational sessions explaining the relative risks and benefits of opioids, while offering alternative analgesic modalities. Increasing education to change pain-management culture will ultimately need to be carried out on a large scale to create population-level change.

Finally, our immediate future plan is to complete enrollment for this present study. Once this single-institution investigation is complete, we will analyze our results. Provided this study demonstrates safety and efficacy, we will launch a population health study with protocol implementation across a large, state-wide healthcare system.

\section{Summary and conclusions}

We describe the detailed protocols used in the first multispecialty orthopedic RCT comparing totally opioid-free perioperative pain management to traditional analgesic pathways. This represents an initial step towards demonstrating that non-opioid multimodal protocols can provide a safe and predictable pathway for patients undergoing elective surgery. Our goal in reporting these pathways is so that they may be implemented at other institutions and utilized in the nationwide fight against the opioid epidemic.

\section{Abbreviations}

ACDA: Anterior cervical disc arthroplasty; ACDF: Anterior cervical discectomy and fusion; CMC: Carpometacarpal; $\mathrm{CrCl}$ : Creatinine clearance; NNT: Number needed to treat; NRS: Numeric rating scale; NSAIDs: Non-steroidal anti- 
inflammatories; RCT: Randomized controlled trial; RTSA: Reverse total shoulder arthroplasty; THA: Total hip arthroplasty; TSA: Total shoulder arthroplasty

\section{Acknowledgements}

The authors would like to thank Susan Odum, PhD for her assistance with statistical planning and analysis; and the following Sub-specialty leaders for their involvement on the steering committee to develop the analgesic protocols tested in the study: Nady Hamid, MD (OrthoCarolina Shoulder \& Elbow), Daniel Leas, MD (Atrium Health Orthopedic Resident), Todd Chapman, MD (OrthoCarolina Spine), Todd Irwin, MD (OrthoCarolina Foot \& Ankle), Bryan Loeffler, MD (OrthoCarolina Hand \&Wrist), James Fleischli, MD (OrthoCarolina Sports Medicine), Walter Beaver, MD (OrthoCarolina Hip \& Knee), John Camp, MD (Atrium Health Anesthesiology), Kathryn Chance, MD (Atrium Health Anesthesiology), Sean McCaffrey, CRNA, MSN (Atrium Health Anesthesiology), Dan Briggs, MD (Novant Health Anesthesiology).

\section{Authors' contributions}

We adhered to the ICMJE authorship criteria. ES drafted this work and contributed substantial edits and revisions. DL contributed to the design of the protocol reported in this work. CM was the project manager of the study and contributed to the design of the protocol. MW contributed substantial edits and revisions. $\mathrm{NH}$ made substantial contributions to the conception and design of the reported study. $\mathrm{NH}$ is the primary investigator and sponsor for the clinical trial. All authors have reviewed and approved this manuscript for submission.

\section{Funding}

This study was supported by a Duke Endowment Grant. The foundation was not involved in any critical portion of the protocol, including the study design, data collection, data management, or data analysis.

The foundation did conduct an external review of the protocol. After meeting grant requirements, the grant proposal and protocol were reviewed in their entirety by the Duke Endowment Health Care Program Officer (Stacy Warren). Grants were narrowed and reviewed by the Duke Endowment Director of Health Care. A select group were selected for site visits. The Health Care Program Officer met with the grantee to discuss specific strategies for implementation and sustainability. The Officer then represented the Grantee at the Board of Trustee meeting. The Board reviewed and discussed the grant application prior to voting on the final recipient to receive funding support. Contact Information: Stacy Warren, Program Officer for Health Care, Duke Endowment, swarren@tde.org

\section{Availability of data and materials}

The study corresponding to this manuscript is still in the recruitment phase. Therefore, no available data currently exists to be shared. However, once the study has entered the data reporting phase, the datasets used and/or analysed during the current study will be available from the corresponding author on reasonable request. The protocol and results will be released onto clinicaltrials.gov as is required upon study completion. There are no contractual agreements limiting investigator access to study data.

\section{Ethics approval and consent to participate}

This study received ethics approval by two local institutional review boards: Chesapeake IRB and Presbyterian Healthcare IRB (operated by Novant Health). These approvals covered all sites involved in the study. Eligible patients were approached for informed consent by the physician and research staff. Patients watched an introductory video explaining the purpose of the clinical trial. The physician also led an in-depth preoperative discussion explaining each approach to analgesia (opioid vs. opioid-free) and postoperative expectations. Consent was either then obtained for the study, or if patients declined to participate in the randomized trial, they were presented with the option of enrolling in the observational group where they would be able to select either the control opioid-containing arm or the opioid-free arm. There were no restrictions on patient care due to enrollment in the study.

\section{Consent for publication}

Not applicable.

\section{Competing interests}

The authors declare that they have no competing interests.

\section{Author details}

'Carolinas Medical Center, Atrium Health Musculoskeletal Institute, P.O. Box 32861, Charlotte, NC 28232, USA. ${ }^{2}$ Carolina Neurosurgery and Spine Associates, Charlotte, NC, USA. ${ }^{3}$ OrthoCarolina Research Institute, Charlotte, NC, USA. ${ }^{4}$ OrthoCarolina Shoulder and Elbow Center, Charlotte, NC, USA.

Received: 30 September 2020 Accepted: 11 January 2021

Published online: 23 January 2021

\section{References}

1. Ferry N, Dhanjal S. Opioid Anesthesia. [Updated 2020 Aug 16]. In: StatPearls [Internet]. Treasure Island: StatPearls Publishing; 2020. Available from: https://www.ncbi.nlm.nih.gov/books/NBK532956/.

2. Fletcher D, Martinez V. Opioid-induced hyperalgesia in patients after surgery: a systematic review and a meta-analysis. Br J Anaesth. 2014;112(6): 991-1004.

3. Lee HJ, Yeomans DC. Opioid induced hyperalgesia in anesthetic settings. Korean J Anesthesiol. 2014;67(5):299-304.

4. McKeown JL. Pain management issues for the geriatric surgical patient. Anesthesiol Clin. 2015;33(3):563-76.

5. National Academies of Sciences, Engineering, and Medicine; Health and Medicine Division; Board on Health Sciences Policy; Committee on Pain Management and Regulatory Strategies to Address Prescription Opioid Abuse. Pain Management and the Opioid Epidemic: Balancing Societal and Individual Benefits and Risks of Prescription Opioid Use. In: Phillips JK, Ford MA, Bonnie RJ, editors. 4, Trends in Opioid Use, Harms, and Treatment. Washington (DC): National Academies Press (US); 2017. Available from: https://www.ncbi.nlm.nih.gov/books/NBK458661/,

6. Macintyre PE, et al. Costs and consequences: a review of discharge opioid prescribing for ongoing management of acute pain. Anaesth Intensive Care. 2014;42(5):558-74

7. Bhashyam AR, Keyser C, Miller CP, Jacobs J, Bluman E, Smith JT, Chiodo C. Prospective evaluation of opioid use after adoption of a prescribing guideline for outpatient foot and ankle surgery. Foot Ankle Int. 2019;40(11): 1260-6. https://doi.org/10.1177/1071100719863711.

8. Bicket MC, Long JJ, Pronovost PJ, Alexander GC, Wu CL. Prescription opioid analgesics commonly unused after surgery: a systematic review. JAMA Surg. 2017;152(11):1066-71. https://doi.org/10.1001/jamasurg.2017.0831.

9. Gardner V, Gazzaniga D, Shepard M, Grumet R, Rubin B, Dempewolf M, et al. Monitoring postoperative opioid use following simple arthroscopic Meniscectomy: a performance-improvement strategy for prescribing recommendations and community safety. JB JS Open Access. 2018;3(4): e0033. https://doi.org/10.2106/jbjs.oa.18.00033.

10. Kim N, Matzon JL, Abboudi J, Jones C, Kirkpatrick W, Leinberry CF, et al. A prospective evaluation of opioid utilization after upper-extremity surgical procedures: identifying consumption patterns and determining prescribing guidelines. J Bone Joint Surg Am. 2016;98(20):e89. https://doi.org/10.2106/ jbjs.15.00614.

11. Sabatino MJ, Kunkel ST, Ramkumar DB, Keeney BJ, Jevsevar DS. Excess opioid medication and variation in prescribing patterns following common Orthopaedic procedures. J Bone Joint Surg Am. 2018;100(3):180-8. https:// doi.org/10.2106/jbjs.17.00672.

12. Saini S, McDonald EL, Shakked R, Nicholson K, Rogero R, Chapter M, et al. Prospective evaluation of utilization patterns and prescribing guidelines of opioid consumption following orthopedic foot and ankle surgery. Foot Ankle Int. 2018;39(11):1257-65. https://doi.org/10.1177/1071100718790243.

13. National Institute on Drug. Overdose Death Rates. Published March 10, 2020. Accessed 1 May 2020. https://www.drugabuse.gov/related-topics/ trends-statistics/overdose-death-rates

14. Seib RK, Paul JE. Preoperative gabapentin for postoperative analgesia: a meta-analysis. Can J Anaesth. 2006:53(5):461-9.

15. Doleman $B$, et al. A systematic review and meta-regression analysis of prophylactic gabapentin for postoperative pain. Anaesthesia. 2015;70(10): 1186-204.

16. Mishriky BM, Waldron NH, Habib AS. Impact of pregabalin on acute and persistent postoperative pain: a systematic review and meta-analysis. $\mathrm{Br} \mathrm{J}$ Anaesth. 2015;114(1):10-31.

17. Banerjee $P$, Rogers BA. Systematic review of high-volume multimodal wound infiltration in total knee arthroplasty. Orthopedics. 2014;37(6):403-12.

18. Marques EM, et al. Local anaesthetic infiltration for peri-operative pain control in total hip and knee replacement: systematic review and meta- 
analyses of short- and long-term effectiveness. BMC Musculoskelet Disord. 2014;15:220.

19. Skolnik A, Gan TJ. New formulations of bupivacaine for the treatment of postoperative pain: liposomal bupivacaine and SABER-bupivacaine. Expert Opin Pharmacother. 2014;15(11):1535-42.

20. Wick EC, Grant MC, Wu CL. Postoperative multimodal analgesia pain management with nonopioid analgesics and techniques: a review. JAMA Surg. 2017;152(7):691-7. https://doi.org/10.1001/jamasurg.2017.0898.

21. Frauenknecht J, Kirkham KR, Jacot-Guillarmod A, Albrecht E. Analgesic impact of intra-operative opioids vs. opioid-free anaesthesia: a systematic review and meta-analysis. Anaesthesia. 2019;74(5):651-62. https://doi.org/10. 1111/anae.14582.

22. Soffin EM, Wetmore DS, Beckman JD, et al. Opioid-free anesthesia within an enhanced recovery after surgery pathway for minimally invasive lumbar spine surgery: a retrospective matched cohort study. Neurosurg Focus. 2019;46(4):E8. https://doi.org/10.3171/2019.1.FOCUS18645.

23. Tedesco D, Gori D, Desai KR, et al. Drug-free interventions to reduce pain or opioid consumption after total knee arthroplasty: a systematic review and meta-analysis. JAMA Surg. 2017;152(10):e172872. https://doi.org/10.1001/ jamasurg.2017.2872.

24. Chughtai M, Sodhi N, Jawad M, et al. Cryotherapy treatment after Unicompartmental and Total knee Arthroplasty: a review. J Arthroplasty. 2017;32(12):3822-32. https://doi.org/10.1016/j.arth.2017.07.016.

25. De Oliveira GS, Almeida MD, Benzon HT, McCarthy RJ. Perioperative single dose systemic dexamethasone for postoperative pain: a meta-analysis of randomized controlled trials. Anesthesiology. 2011;115(3):575-88. https://doi. org/10.1097/ALN.0b013e31822a24c2.

26. Waldron NH, Jones CA, Gan TJ, Allen TK, Habib AS. Impact of perioperative dexamethasone on postoperative analgesia and side-effects: systematic review and meta-analysis. Br J Anaesth. 2013;110(2):191-200. https://doi.org/ 10.1093/bja/aes431.

27. Kazerooni R, Tran MH. Evaluation of Celecoxib addition to pain protocol after Total hip and knee Arthroplasty stratified by opioid tolerance. Clin J Pain. 2015;31(10):903-8.

28. Khan JS, et al. Preoperative celecoxib in noncardiac surgery: a systematic review and meta-analysis of randomised controlled trials. Eur J Anaesthesiol. 2016;33(3):204-14.

29. Koh W, Nguyen KP, Jahr JS. Intravenous non-opioid analgesia for peri- and postoperative pain management: a scientific review of intravenous acetaminophen and ibuprofen. Korean J Anesthesiol. 2015;68(1):3-12.

30. Nir RR, et al. Preoperative preemptive drug administration for acute postoperative pain: a systematic review and meta-analysis. Eur J Pain. 2016; 20(7):1025-43

31. Rouhani A, et al. Effects of preoperative non-steroidal anti-inflammatory drugs on pain mitigation and patients' shoulder performance following rotator cuff repair. Adv Pharm Bull. 2014;4(4):363-7.

32. Ilyas AM, Miller AJ, Graham JG, Matzon JL. A prospective, randomized, double-blinded trial comparing acetaminophen, ibuprofen, and oxycodone for pain management after hand surgery. Orthopedics. 2019;42(2):110-5. https://doi.org/10.3928/01477447-20190221-02.

33. Pham H, Pickell M, Yagnatovsky M, et al. The Utility of Oral Nonsteroidal Antiinflammatory Drugs Compared with Standard Opioids Following Arthroscopic Meniscectomy: A Prospective Observational Study. Arthroscopy. 2019;35(3): 864-870.e1. https://doi.org/10.1016/j.arthro.2018.09.018.

34. Thybo KH, Hägi-Pedersen D, Dahl JB, et al. Effect of combination of Paracetamol (acetaminophen) and ibuprofen vs either alone on patientcontrolled morphine consumption in the first 24 hours after Total hip Arthroplasty: the PANSAID randomized clinical trial. JAMA. 2019;321(6):56271. https://doi.org/10.1001/jama.2018.22039.

35. Richards D. The Oxford pain group league table of analgesic efficacy. Evid Based Dent. 2004;5(1):22-3. https://doi.org/10.1038/sj.ebd.6400237.

36. Leas DP, Connor PM, Schiffern SC, D'Alessandro DF, Roberts KM, Hamid N. Opioid-free shoulder arthroplasty: a prospective study of a novel clinical care pathway. J Shoulder Elbow Surg. 2019;28(9):1716-22. https://doi.org/10. 1016/j.jse.2019.01.013.

37. Eckhard L, Jones $\mathrm{T}$, Collins JE, Shrestha S, Fitz W. Increased postoperative dexamethasone and gabapentin reduces opioid consumption after total knee arthroplasty. Knee Surg Sports Traumatol Arthrosc. 2019:27(7):2167-72. https://doi.org/10.1007/s00167-019-05449-8.

38. Pearson AC, Moman RN, Moeschler SM, Eldrige JS, Hooten WM. Provider confidence in opioid prescribing and chronic pain management: results of the opioid therapy provider survey. J Pain Res. 2017;10:1395-400. https:// doi.org/10.2147/JPR.S136478.

39. Cordts GA, Grant MS, Brandt LE, Mears SC. A qualitative and quantitative needs assessment of pain management for hospitalized orthopedic patients. Orthopedics. 2011;34(8):e368-73. https://doi.org/10.3928/0147744720110627-11.

40. Kaafarani HMA, Eid Al, Antonelli DM, et al. Description and impact of a comprehensive multispecialty multidisciplinary intervention to decrease opioid prescribing in surgery. Ann Surg. 2019;270(3):452-62. https://doi.org/ 10.1097/SLA.0000000000003462.

41. McCalmont JC, Jones KD, Bennett RM, Friend R. Does familiarity with CDC guidelines, continuing education, and provider characteristics influence adherence to chronic pain management practices and opioid prescribing? J Opioid Manag. 2018;14(2):103-16. https://doi.org/10.5055/jom.2018.0437.

42. Yorkgitis BK, Paffett C, Brat GA, Crandall M. Effect of surgery-specific opioidprescribing education in a safety-net hospital. J Surg Res. 2019;243:71-4. https://doi.org/10.1016/j.jss.2019.05.003.

43. Andelman SM, Bu D, Debellis N, et al. Preoperative patient education may decrease postoperative opioid use after Meniscectomy. Arthrosc Sports Med Rehabil. 2020;2(1):e33-8. https://doi.org/10.1016/j.asmr.2019.10.006.

44. Farley KX, Anastasio AT, Kumar A, Premkumar A, Gottschalk MB, Xerogeanes J. Association between quantity of opioids prescribed after surgery or preoperative opioid use education with opioid consumption. JAMA. 2019; 321(24):2465-7. https://doi.org/10.1001/jama.2019.6125.

45. Rucinski K, Cook JL. Effects of preoperative opioid education on postoperative opioid use and pain management in orthopaedics: a systematic review. J Orthop. 2020;20:154-9. https://doi.org/10.1016/j.jor. 2020.01.020.

46. Syed UAM, Aleem AW, Wowkanech C, et al. Neer award 2018: the effect of preoperative education on opioid consumption in patients undergoing arthroscopic rotator cuff repair: a prospective, randomized clinical trial. J Shoulder Elbow Surg. 2018;27(6):962-7. https://doi.org/10. 1016/j.jse.2018.02.039

47. von Eisenhart Rothe A, Zenger M, Lacruz ME, et al. Validation and development of a shorter version of the resilience scale RS-11: results from the population-based KORA-age study. BMC Psychol. 2013;1:25. https://doi. org/10.1186/2050-7283-1-25.

48. Harris PA, Taylor R, Thielke R, Payne J, Gonzalez N, Conde JG. Research electronic data capture (REDCap)--a metadata-driven methodology and workflow process for providing translational research informatics support. J Biomed Inform. 2009;42(2):377-81. https://doi.org/10.1016/j.jbi.2008.08.010.

49. Breivik $\mathrm{H}$, Borchgrevink $\mathrm{PC}$, Allen SM, et al. Assessment of pain. Br J Anaesth. 2008;101(1):17-24. https://doi.org/10.1093/bja/aen103.

50. Richards RR, An KN, Bigliani LU, Friedman RJ, Gartsman GM, Gristina AG, lannotti JP, Mow VC, Sidles JA, Zuckerman JD. A standardized method for the assessment of shoulder function. J Shoulder Elbow Surg. 1994;3(6):34752. https://doi.org/10.1016/S1058-2746(09)80019-0 Epub 2009 Feb 13.

51. Martin RL, Irrgang JJ, Burdett RG, Conti SF, Van Swearingen JM. Evidence of validity for the foot and ankle ability measure (FAAM). Foot Ankle Int. 2005; 26(11):968-83. https://doi.org/10.1177/107110070502601113.

52. Baker DW. History of the joint Commission's pain standards: lessons for Today's prescription opioid epidemic. JAMA. 2017;317(11):1117-8. https:// doi.org/10.1001/jama.2017.0935.

53. Scher C, Meador L, Van Cleave JH, Reid MC. Moving beyond pain as the fifth vital sign and patient satisfaction scores to improve pain care in the 21st century. Pain Manag Nurs. 2018;19(2):125-9. https://doi.org/10.1016/j. pmn.2017.10.010

\section{Publisher's Note}

Springer Nature remains neutral with regard to jurisdictional claims in published maps and institutional affiliations. 